\title{
MODELING THE EXTENSIBILITY OF THE SALES PROCESS - SAP S/4HANA APPLICATION
}

\author{
Claudiu BRANDAS \\ West University of Timisoara \\ claudiu.brandas@e-uvt.ro \\ Mihaela MUNTEAN \\ West University of Timisoara \\ mihaela.muntean@e-uvt.ro \\ Otniel DIDRAGA \\ West University of Timisoara \\ otniel.didraga@e-uvt.ro \\ Vlad MANESCU \\ West University of Timisoara \\ vlad.g.manescu@gmail.com
}

\begin{abstract}
Currently, in companies, the sales and marketing departments need qualified information to allow them to accelerate productivity and streamline the sales process. There is also a need for relevant conversation, well-defined target groups, and client interaction using any means of communication. Customizing applications and software plays an essential role for key-users; it enhances human-technology interaction and adapts the solution to legal requirements. We present the extensibility of the sales process in the ERP SAP S/4HANA Cloud System, considering the legal requirements and following step-by-step the stages that a keyuser has to go through when he needs to extend the sales process. The result is an extended sales process with the same, unchanged structure, no matter what future updates the ERP will have.
\end{abstract}

Keywords: extensibility, ERP, processes, cloud computing

JEL classification: M15, L86

DOI: $10.12948 / \mathrm{ie} 2019.04 .16$

\section{Introduction}

Cloud computing is no longer dedicated solely to IT companies. Now, many small and medium-sized organizations have begun to discover the benefits of cloud applications. Industry experts argue that cloud computing allows their organizations to run their applications faster, with a higher level of control, and allows IT professionals to adjust their processes quickly and more efficiently.

We can identify multiple types of cloud-applicable elements such as scalable use, chatbots, business processes, data backup and recovery, application development and testing, data analysis, and social networking [1].

A Cloud-based ERP software comes in the form of a SaaS (Software as a Service). It is maintained in the provider's infrastructure, and the software vendor controls all data created in this system. A client can access the ERP system through a web browser [1].

"Through SAP S/4HANA (SAP Business Suite 4 SAP HANA), Cloud Edition, SAP offers a new generation of business applications through software for Big Data and agility" [2]. 
"SAP S/4HANA Cloud is provided in 3 versions: Cloud Marketing Edition, Cloud Enterprise Edition, and Cloud Project Services Edition" [2].

SAP S/4HANA Cloud Enterprise Edition provides a comprehensive set of scenarios for an organization, including financial, logistics, and supply. Among these, we can list certain app collections that are grouped into business catalogs such as: "Order to Cash: Accounts Receivable", "Collaborative Invoice to Pay: Accounts Payable", "Cash Management", "Profitability and Cost Analysis", "Order to Cash: Sell from Stock", and "Plan to Procure" [2].

\section{Software extensibility}

Software processes can be configured and adapted, considering the organization needs and project-specific contexts, in an environment where software flexibility helps reuse, modify, and extend the existing business processes [3].

In this respect, the S/4HANA Cloud comes with a new concept called "Extensibility". An important pillar of this concept is the set of tools that is part of S/4HANA. The application suite for key users follows the cloud services principle. They are "built for the cloud, but they are also available in on premise editions: they are completely web-based (developed using Fiori principles and technologies), and they are designed for key users, the artifacts created with the key user application set are life-stable" [4]. The applications are based on "stable public APIs so they can continue to operate regardless of the ERP update cycles" [4].

Key users can "change the schema of tables and forms directly into the SAP S/4HANA Cloud ERP application interface" in "UI Adaptation Mode" mode. Thus, the user is able to: "hide fields in a form, table, or filter, hide application groups, rename field labels, add new fields to the interface, move a field or a group of fields, combine multiple fields in one line, define filters and variations for a table" [4].

In the "Custom Fields and Logic" App, the key user can add customized new fields. The available features are: Creating and editing custom fields; Deleting custom fields; Controlling the use of custom fields in the interface, reports, forms for emails, services, processes, and searches; Defining association fields to a custom business object or standard business object [4], [5].

With Fiori "Custom Business Objects", the key user can "create and control custom business objects" [4]. A custom business object is "a hierarchical set of tables in the database, with an API for creating, reading, updating, and deleting data" [4]. The user can add logic in a web editor using the cloud-based version of the ABAP language [4].

The "Custom Business Objects" App provides the following options [4]: "Create business objects and tables in the corresponding database; Adding fields to a business object, deleting fields and deleting business objects that were not transferred into the production system; Create CDS views, OData services, and user interfaces to add data to business object; Create multiple sub-nodes for a business object; Define association fields to another custom business object; Implementing logic at node level; Writing custom business objects from custom logic".

\section{Case study - implementing a "Business Place" extension to the sales process 3.1. Business analysis and scenarios}

The application is the extension of a business scenario for a particular country, adding the concept of "Business Place".

In some countries, at start-up, corporations are required to obtain a unique identification code (VAT registration number) from the tax authorities for each site that is used for all types of tax reporting [6], [7].

A "Business Place" is a part of a company under the jurisdiction of a tax office. Software to help customers who issue invoices and file tax returns should include the following: Business 
Place definition, and keeping the information at the Business Place level. Also, this software should allow data manipulation at the Business Place level during business processes. An organizational unit (business unit) that is registered with tax authorities to report sales or purchase taxes is a Business Place [6], [7].

An organizational unit must be associated with a "Business Place". A "Business Place" can be associated with several organizational units. This Custom Business Object must be available in the following transactional interfaces, and its value is transferred between processes: Orders - "Manage Sales Orders" App; Tax invoices to customers - "Manage Billing Documents" App; Delivery Documents - "Manage Outbound Deliveries" App; Logbook - "Manage Journal Entries" App.

For the field value to be transferred, we need to enable the field in two business scenarios: "Sales Document to Billing Document on header level" and "Sales Document to Delivery on header level".

\subsection{Software design}

Best practices in designing and developing Custom Business Objects imply the following process steps (figure 1).

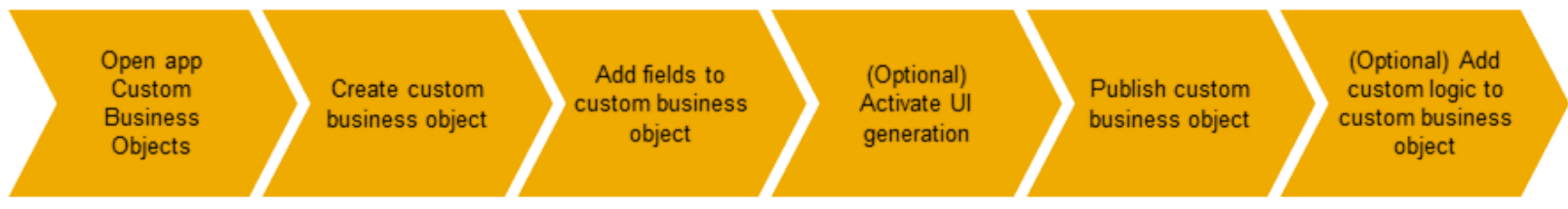

Figure 1. Process steps to create a Custom Business Object [8]

Based on the above approach, in this paper, we created a custom Business Configuration Object with the database structure presented in figure 2 .
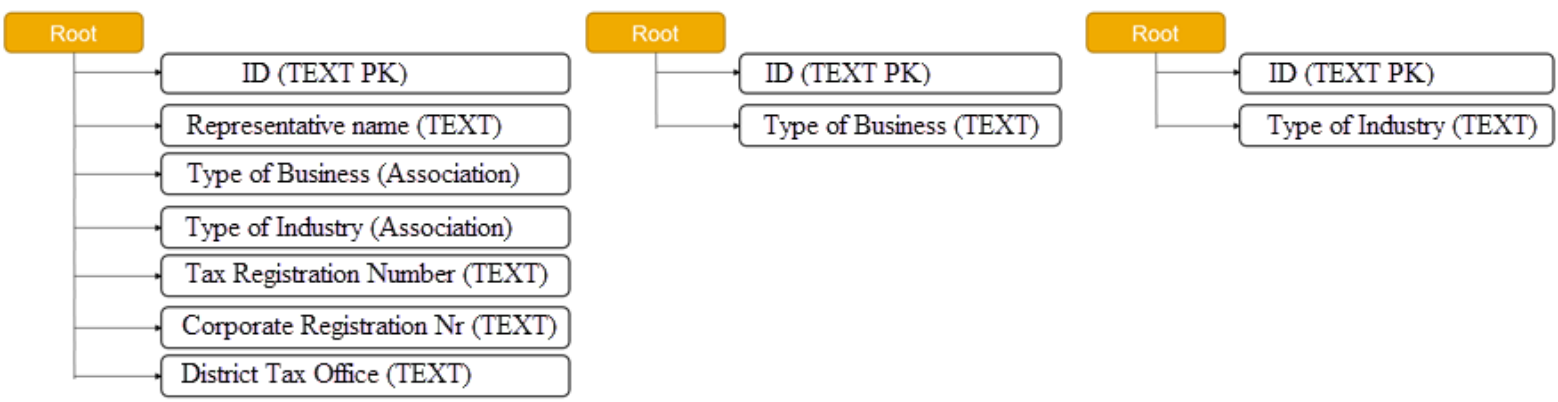

Figure 2. The database structure for the "Business Place", "Type of Business", and "Type of Industry" Business Objects [own development]

The interfaces for orders, invoices, delivery, and general ledger, are part of the standard system functionality and do not need to be generated. They will be extended with a field that will be associated with the "Business Place" Business Object.

The prototype of the interface associated with the Business Object is presented in figure 3. 
www.conferenceie.ase.ro

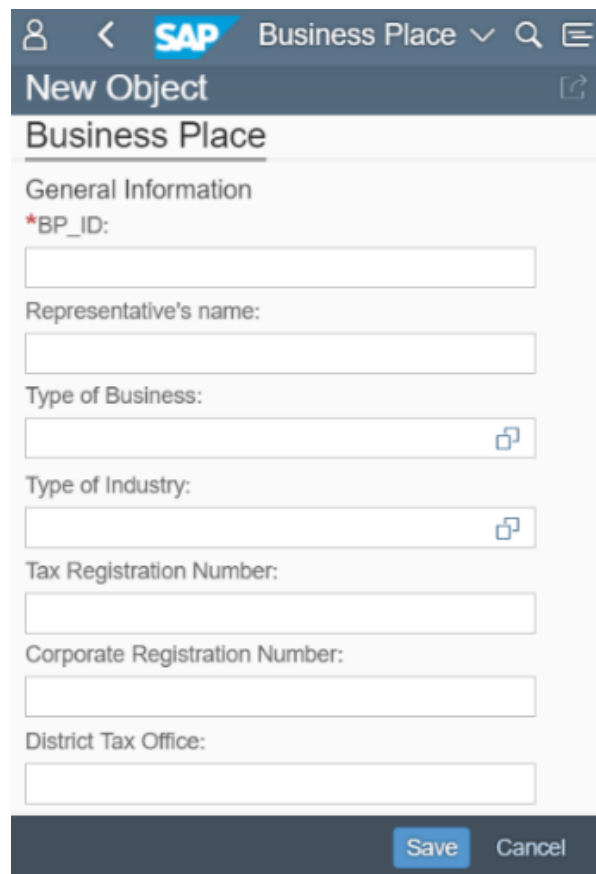

Figure 3. Interface prototype for the "Business Place" Custom Business Object [own development]

\subsection{Software implementation and testing}

The software architect and the client will choose a development system that can be accessed through a link similar to "https://my*******.s4hana.ondemand.com". The development and testing will be done in this system, and when the application is complete, it will be transported into a productive system. Also, the software architect must create appropriate users and roles in the system. To access the "Extensibility" catalog applications, as well as to access the desired business contexts for the sales process, the user needs specific roles in the S/4HANA Cloud system [9]. The roles are presented in Table 1.

\begin{tabular}{|c|c|c|c|}
\multicolumn{4}{|c|}{ Table 1. Roles and business catalogs [own development] } \\
\hline Roles & Business Catalog & $\begin{array}{c}\text { Catalog } \\
\text { Description }\end{array}$ & Used Applications \\
\hline SAP_BC_CORE_EXT & SAP_BC_CORE_EXT & Extensibility & $\begin{array}{l}\text { Custom Fields and Logic, } \\
\text { Custom Business Objects }\end{array}$ \\
\hline
\end{tabular}

Creating the "Business Place", "Type of Industry", and "Type of Business" Business Objects starts with the Custom Business Objects application in the Extensibility catalog, as shown in figure 4 . The next step is for the user to specify the data types for each field. 


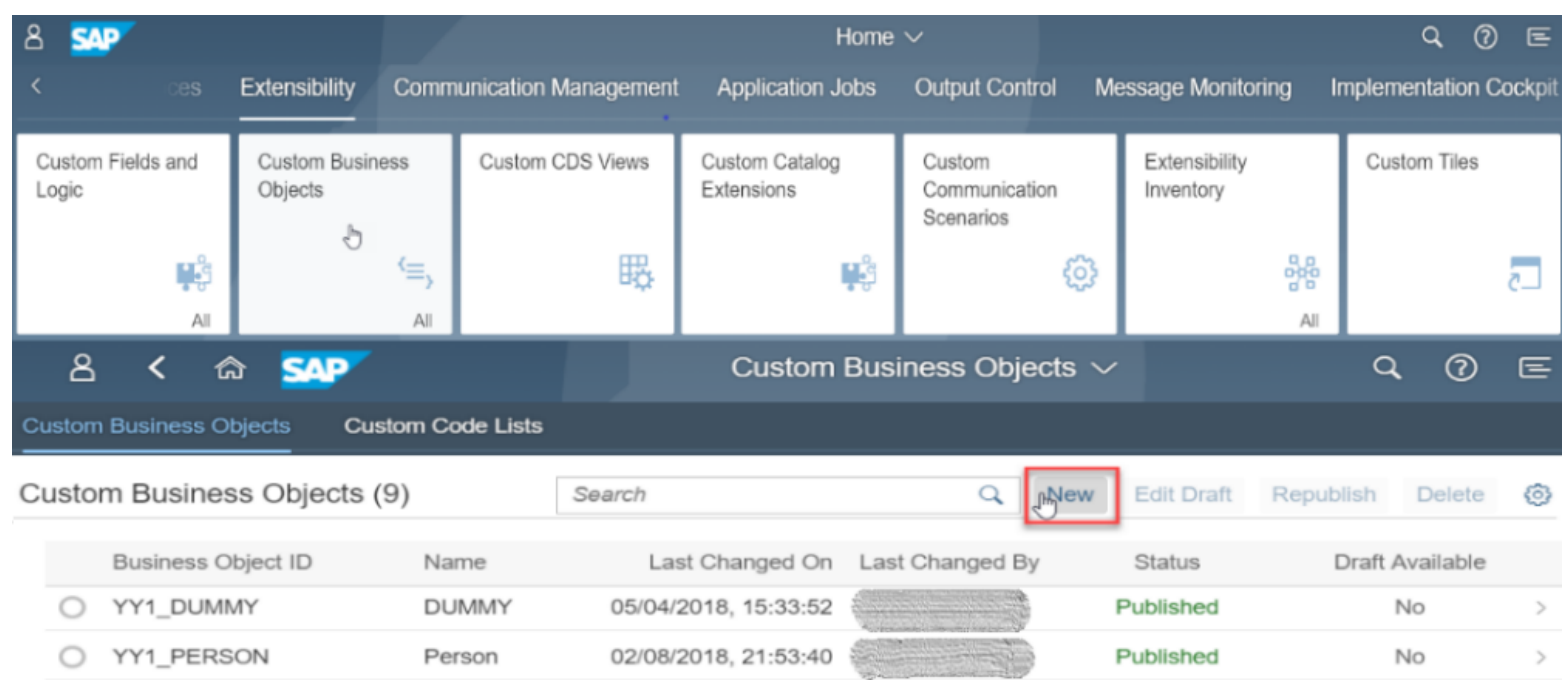

Figure 4. Adding a new Custom Business Object [own development]

Business Object Interfaces are generated to allow users to add records to smart tables. After this stage has been completed, it is necessary to create a Custom Field named "Business Place" that we will use further in the documents associated with the sales process. The field is created using the Custom Fields and Logic App from the Extensibility catalog, and we need to select the Business Context, the label, and field type, as shown in figure 5.

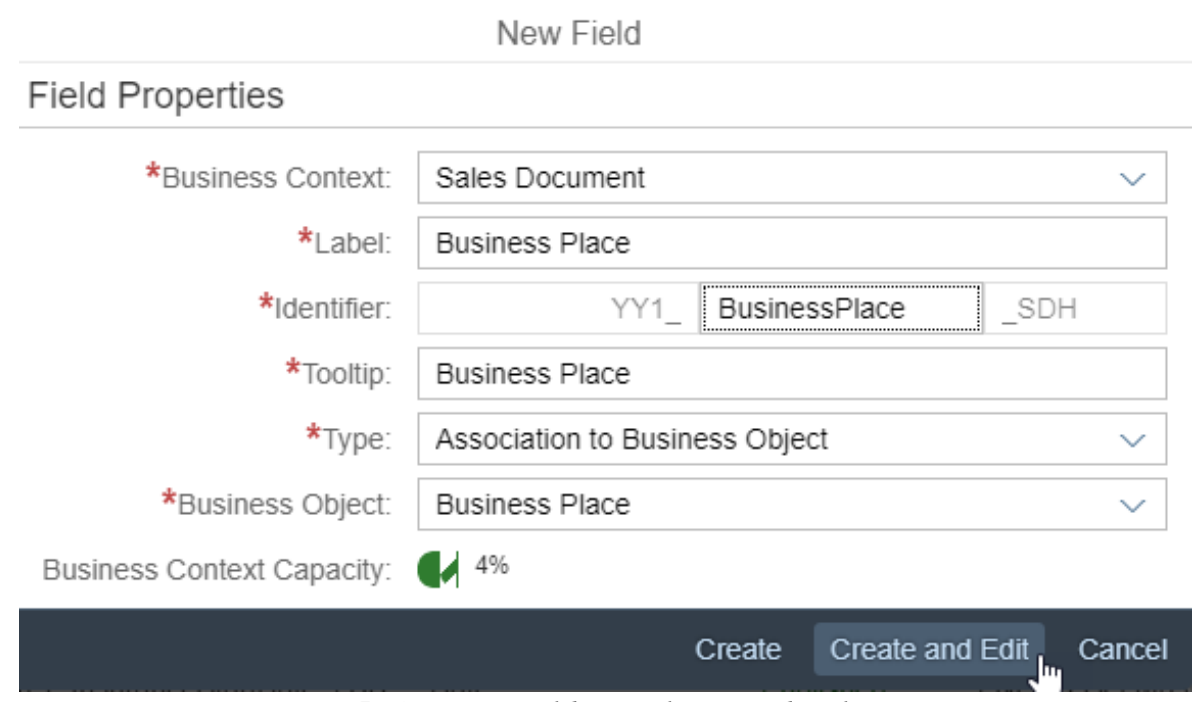

Figure 5. Custom Field Details [own development]

For the field value to propagate from one interface to another, it is recommended to enable the field in the following scenarios: "Sales Document to Billing Document on Header Level"; "Sales Documentation to Delivery on Header Level"; "Billing Document to Journal Entry on Item Level" [10], as shown in figure 6. After enabling the field in the business scenarios and the appropriate interfaces, it is available for testing by S/4HANA Cloud Edition testers, and it will be tested by key application users. 


\begin{tabular}{|c|c|c|c|}
\hline $8<$ ก & \multicolumn{2}{|c|}{ Custom Fields and Logic $\vee$} & E \\
\hline \multicolumn{4}{|l|}{ Sales: Billing Document } \\
\hline General Information Uls and Reports (8) Email Templates (1) Form Templates (53) & Business Scenarios (6): & OData APIs (2) & SOAP APIs (1) \\
\hline \multicolumn{4}{|l|}{ Scenarios Available for Business Context Sales: Billing Document (6) } \\
\hline Description & \multicolumn{3}{|l|}{ Field Usage } \\
\hline > Billing Document to Pricing Communication on Header Level & Disabled & Enable Usage & \\
\hline > Billing Document to Sales Document on Header Level & Disabled & Enable Usage & \\
\hline > Delivery to Billing Document from Item to Header Level & Disabled & Enable Usage & \\
\hline > Delivery to Billing Document on Header Level & Disabled & Enable Usage & \\
\hline > Sales Document to Billing Document from Item to Header Level & Disabled & Enable Usage & \\
\hline \multicolumn{4}{|l|}{ All Scenarios Selected for this Field (3) } \\
\hline Description & \multicolumn{3}{|l|}{ Field Usage } \\
\hline > Billing Document to Journal Entry on Item Level & \multicolumn{3}{|l|}{ Enabled } \\
\hline > Sales Document to Billing Document on Header Level & \multicolumn{3}{|l|}{ Enabled } \\
\hline > Sales Document to Delivery on Header Level & \multicolumn{3}{|l|}{ Enabled } \\
\hline
\end{tabular}

Once test data has been added to the Type of Business and Type of Industry tables, data can be added to the Business Place table. Data will be added by accessing the Business Object interface via the "Go to Generated UI" link.

To test the created Business Object, we used the "Sales Document to Billing Document on Header Level" scenario. The flow for this scenario includes the Sales Order creation; Adding the "Business Place" field on the sales order; Adding values to the "Business Place" field; Invoice Creation (Billing Document) containing the "Business Place" field [10].

Next, we created the Outbound Delivery, using the "Create Outbound Deliveries with Order Reference" App in the "Outbound Delivery Processing" catalog. After the creation of the delivery, we check if the "Business Place" field has the same content as the order which the delivery was based on. Considering that the delivery was created without any warnings or errors, the user will be able to move to the final step of the process, namely the creation of the invoice using the "Create Billing Documents" application, in the "Billing Documents" catalog. Before issuing the invoice, the key user will check the customer details and the contents of the Business Place field that has been retrieved from the delivery. If this data is valid, the user can issue the invoice by clicking the "Post Billing Document" button, as shown in figure 7.

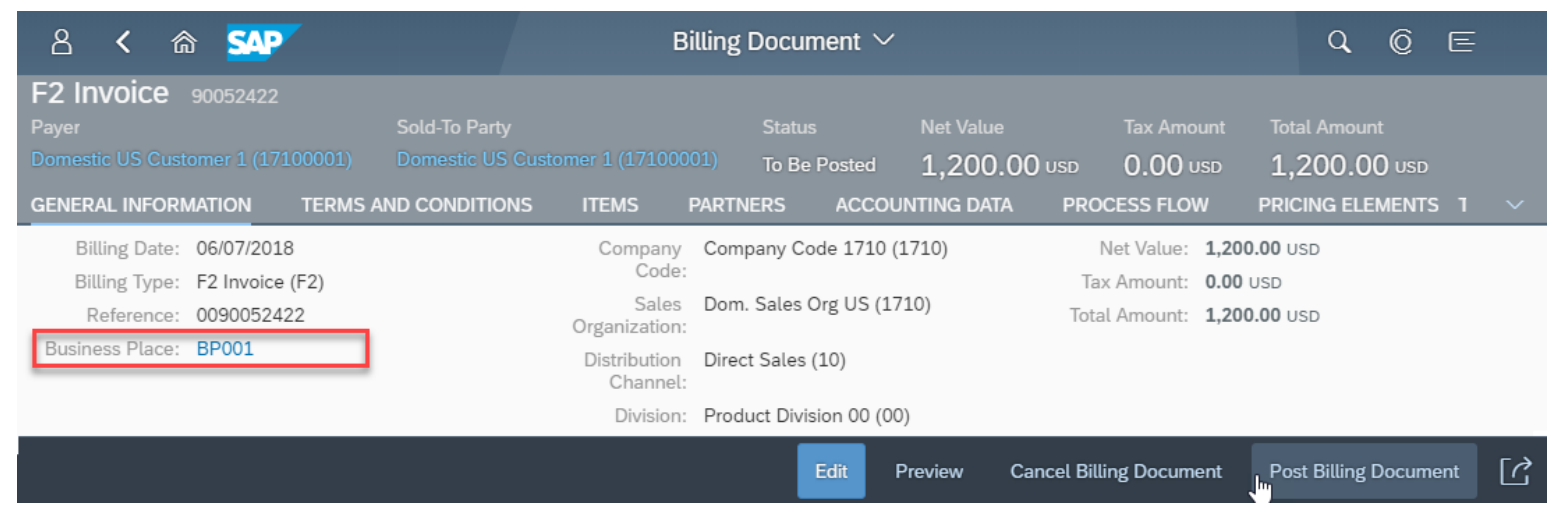

Figure 7. Billing Document using the extension [own development]

Once the invoice is issued, the user will decide whether the process was executed correctly, and the content of the Business Place field has been passed from the predecessor document to the successor document. The flow of documents in which the Business Place field was created and validated is shown in figure 8 . 


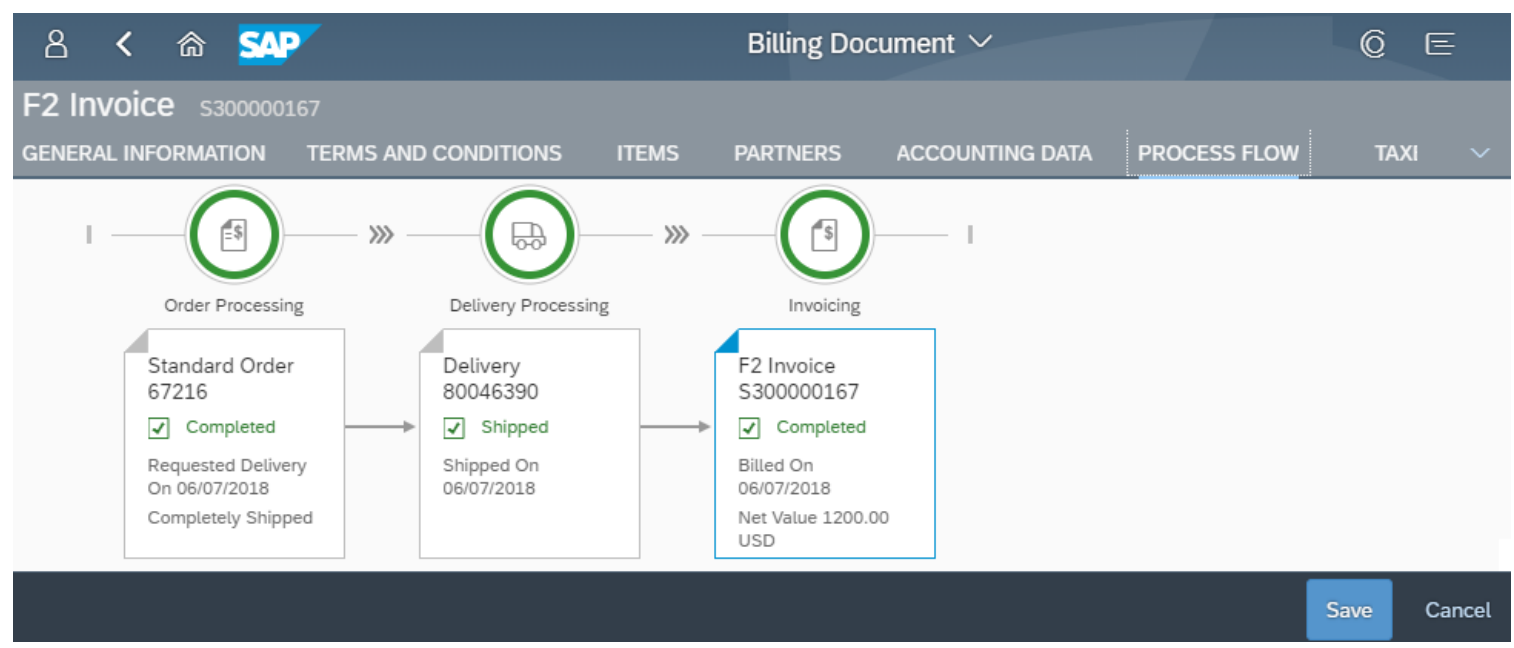

Figure 8. The tested Process-Flow [own development]

\section{Conclusions}

In the paper, we presented the steps to model the extensibility of the sales process, implemented on SAP S/4HANA.

The SAP S4/HANA Cloud solution extensibility apps cover a wide range of topics that let customers adapt standard software to their specific business requirements. For both solution variants (cloud and on-premise), SAP S/4HANA includes a set of applications for key users, which can adapt the solution to their own needs by extending the interface and contexts, creating custom fields and tables, creating and extending analytical reports and forms, and changing business logic by adding logic to the ABAP for Cloud programming language.

The extensibility framework implies the creation of new Custom Business Objects, i.e., "Business Place", Type of Industry", "Type of Business", and the modeling of the corresponding extended process flow.

The advantages of the proposed extensibility are:: end-to-end applications (key users, experts, and implementation consultants can apply changes in the risk-free area), step-by-step development (personalized extensions are not closely related to processes business standard data, processes are required, but the lifetime of the extensions does not depend on the system records), and the help that Cloud Solutions partners receive to apply the extension principles and implement different solutions.

The approach based on Custom Business Objects also has some limits and disadvantages. If a Business Object is modified after it is used elsewhere (reports, forms), there is the risk to alter the data processing. At this moment, Custom Business Objects cannot have attachments; this feature may come with a future release of S/4HANA Cloud.

\section{References}

[1] J. Thomas. It's Time to Swap Your Outdated ERP Software to Cloud-Based ERP. Cloud Zone-Analysis. Internet: https://dzone.com/articles/its-time-to-swap-your-outdated-erpsoftware-to-clo, July 08, 2015 [Feb. 20, 2019].

[2] SAP SE. SAP User Assistance 2015. Internet: https://cp.hana.ondemand.com/dps/d/preview/3f07b8545988b409e10000000a423f68/151 1\%20500/en-US/frameset.htm?7af7b8541486ed05e10000000a4450e5.html, [Feb. 25, 2019].

[3] M. Kuhrmann, T. Ternité, J. Friedrich, A. Rausch, M. Broy, "Flexible software process lines in practice: A metamodel-based approach to effectively construct and manage families of software process models", The Journal of Systems and Software, 121, pp. 49-71, 2016. 
www.conferenceie.ase.ro

[4] T. Schneider. The Key User Extensibility Tools of S/4 HANA. Internet: https://blogs.sap.com/2015/09/30/the-key-user-extensibility-tools-of-s4-hana/, September 30, 2015, Updated May 15, 2018. [Feb 26, 2019].

[5] SAP SE. SAP Documentation $2015 . \quad$ Internet: https://cp.hana.ondemand.com/dps/d/preview/1a93686c176845f0832a2a73221dd90b/160 5\%20500/en-US/frameset.htm?57909455bf7c4fdd8bcf48d76c1eae33.html, [Feb. 25, 2019].

[6] SAP SE. SAP Documentation $2015 . \quad$ Internet: https://help.sap.com/doc/49f7c5536a51204be10000000a174cb4/1610\%20001/enUS/56b8dd54d4c37274e10000000a423f68.html, [Feb. 25, 2019].

[7] SAP SE. SAP Help Portal v. 6.0 EHP8 SP07. Business Place. 2015. Internet: https://help.sap.com/viewer/402e2f30790b4922b4e7ba3cbe552d8d/6.18.07/enUS/6e75d0531d8b4208e10000000a174cb4.html, [Feb. 25, 2019].

[8] SAP SE. SAP Documentation $2015 . \quad$ Internet: https://cp.hana.ondemand.com/dps/d/preview/1a93686c176845f0832a2a73221dd90b/160 5\%20500/en-US/frameset.htm?c703c5b4e6a24d52a928ea54e8ff5e52.html, [Feb. 25, 2019].

[9] SAP SE. SAP Help Portal. General Functions for the Key User-Business Catalogs. Internet: https://help.sap.com/viewer/DRAFT/f544846954f24b9183eddadcc41bdc3b/1908.500/enUS/dd0abf583e0647e5a536b878efeb5143.html?q=business\%20catalogues, [Feb. 26, 2019].

[10] SAP SE. SAP Help Portal. Process Extensibility for Sales and Billing Documents. Internet:

https://help.sap.com/viewer/7b24a64d9d0941bda1afa753263d9e39/1709\%002/en-

US/10b0f186-fef8-43cc-9b0b-b79d43b74993.html, [Feb. 27, 2019]. 